\title{
A NOTE ON STRESS CONCENTRATION AROUND AN ELLIPTIC HOLE IN MICROPOLAR ELASTICITY
}

\author{
ANIMESH BASU
}

(Recelved 24 May 1976)

(Revised 19 July 1976)

\begin{abstract}
Within the scope of Eringen's linearised micropolar theory, this note outlines a solution for the stress concentration around an elliptic hole in an infinite plate under axial tension.
\end{abstract}

\section{Introduction}

In classical linear elasticity theory, the solutions for the stress concentration around circular and elliptic holes are well known [2]. For elastic materials with microstructure, the fundamental system of field equations includes a couple-stress tensor, in addition to the force-stress tensor. Eringen [1] has formulated the tensor equations for elastic materials with microstructure. Using an approach based on stress functions, the problem of stress concentration around a circular hole has been solved [4]. Here, instead of using stress functions we use a method based on the Helmholtz representation of the displacement vector $\vec{u}$, and the micro-rotation vector $\vec{\phi}$, as sums of two fields, one with a scalar and the other a vector potential.

\section{Outline of method}

Following Eringen [1], the basic equations for a micro-polar elastic solid in the absence of inertia forces are

$$
\begin{aligned}
& (\lambda+2 \mu+\kappa) \nabla \nabla \cdot \vec{u}-(\mu+\kappa) \nabla \times \nabla \times \vec{u}+\kappa \nabla \times \vec{\phi}=0, \\
& (\alpha+\beta+\gamma) \nabla \nabla \cdot \vec{\phi}+\gamma \nabla \times \nabla \times \vec{\phi}+\kappa \nabla \times \vec{u}-2 \kappa \vec{\phi}=0 .
\end{aligned}
$$

The constants $\lambda, \mu, \kappa, \alpha, \beta$ and $\gamma$ denote the material constants of the micro-polar elastic solid. Using

$$
\begin{aligned}
& \vec{u}=\nabla u_{0}+\nabla \vec{U}, \\
& \vec{\phi}=\nabla \phi_{0}+\nabla \times \vec{\Phi},
\end{aligned}
$$


subject to conditions

$$
\begin{array}{ll}
\nabla . \vec{U}=0, & \nabla . \vec{\Phi}=0, \\
\phi_{0}=0, & \vec{\phi}=\phi \vec{e}_{3}, \\
l^{2}=\frac{\gamma(\kappa+\mu)}{\kappa(\kappa+2 \mu)}, &
\end{array}
$$

the equations (2.1) reduce to

$$
\begin{aligned}
& \nabla^{4} u_{0}=0 \\
& \nabla^{4} \phi-\frac{1}{l^{2}} \nabla^{2} \phi=0 \\
& \nabla^{6} U-\frac{1}{l^{2}} \nabla^{4} U=0 .
\end{aligned}
$$

We consider now an infinite plate in a state of uniform tension $S$ (along the $x$-direction) disturbed by an elliptical hole of semiaxes $a$ and $b$ parallel to the $x$ - and $y$-axes, which is free from stress. As the boundary of the hole is an ellipse, it is advantageous to use elliptic coordinates $(\xi, \eta)$ given by $x=$ $c \cosh \xi \cos \eta, y=c \sinh \xi \cos \eta$.

As the hole is stress free, and the plate is subjected to uniform tension $S$ at infinity, we have as boundary conditions

$$
\begin{aligned}
& t_{\xi}^{\xi}=t_{\eta}^{\xi}=0 \text { on the hole } \xi=\xi_{0}, \quad m \frac{\xi}{z}=0 \text { on } \xi=\xi_{0}, \\
& t_{\xi}^{\xi}=\frac{S}{2}(1+\cos 2 \eta), \quad t_{\eta}^{\xi}=-\frac{S}{2} \sin 2 \eta \text { at } \xi=\infty,
\end{aligned}
$$

where $m_{Z}^{\xi}$ is the couple-stress and $t_{1}^{k}$ is the stress-tensor.

In elliptic coordinates $(\xi, \eta)$ the general solutions of the equations (2.4) are given by

$$
\begin{aligned}
& u_{0}=\phi_{0}+\sum_{n=1}^{\infty} \phi_{2 n} \cos 2 n \eta \\
& \phi=\sum_{n=1}^{\infty}\left[A_{2 n} e^{-2 n \xi} \sin 2 n \eta+B_{2 n} \operatorname{Gek}_{2 n}\left(\xi,-q_{1}\right) \operatorname{se}_{2 n}\left(\eta,-q_{1}\right)\right], \\
& U=\sum_{n=1}^{\infty}\left[\psi_{2 n} \sin 2 n \eta+C_{2 n} \operatorname{Gek}_{2 n}\left(\xi,-q_{1}\right) \operatorname{se}_{2 n}\left(\eta,-q_{1}\right)\right]
\end{aligned}
$$

where $\operatorname{se}_{2 n}\left(\eta,-q_{1}\right)$ is the periodic Mathieu function of order $2 n$ and $\operatorname{Gek}_{2 n}\left(\xi,-q_{1}\right)$ is a modified Mathieu function involving $K$-Bessel functions [5]. Further, $q_{1}=c^{2} / 4 l^{2}$ and $A_{2 n}, B_{2 n}$, and $C_{2 n}$ are constants which are determined from the boundary conditions. Also 


$$
\begin{aligned}
& \phi_{0}=a_{0} e^{-2 \xi}+c_{0} \xi, \\
& \phi_{2 n}=a_{2 n-2} e^{-(2 n-2) \xi}+a_{2 n} e^{-(2 n+2) \xi}+a_{2 n}^{\prime} e^{-2 n \xi} \quad(n \geqq 1), \\
& \psi_{0}=b_{0} e^{-2 \xi}+c_{0}^{\prime} \xi, \\
& \psi_{2 n}=b_{2 n-2} e^{-(2 n-2) \xi}+b_{2 n} e^{-(2 n+2) \xi}+b_{2 n}^{\prime} e^{-2 n \xi} \quad(n \geqq 1) .
\end{aligned}
$$

The unknown coefficients $a_{2 n}, b_{2 n}, a_{2 n}^{\prime}, b_{2 n}^{\prime}, b_{0}, c_{0}$, etc. are determined from the boundary condition (2.5), by solving an infinite set of linear equations. ${ }^{*}$ The stress components in elliptic coordinates are given by

$$
\begin{aligned}
\frac{c^{2}}{2}(\cosh 2 \xi-\cos 2 \eta)^{2} t_{\xi}^{\epsilon}= & \lambda(\cosh 2 \xi-\cos 2 \eta)\left(\frac{\partial^{2} u_{0}}{\partial \xi^{2}}+\frac{\partial^{2} u_{0}}{\partial \eta^{2}}\right) \\
& +(2 \mu+\kappa)\left[(\cosh 2 \xi-\cos 2 \eta)\left(\frac{\partial^{2} u_{0}}{\partial \xi^{2}}+\frac{\partial^{2} U}{\partial \xi \partial \eta}\right)\right. \\
& \left.-\sinh 2 \xi\left(\frac{\partial u_{0}}{\partial \xi}+\frac{\partial U}{\partial \eta}\right)+\sin 2 \eta\left(\frac{\partial u_{0}}{\partial \eta}-\frac{\partial U}{\partial \xi}\right)\right],
\end{aligned}
$$$$
\frac{c^{2}}{2}(\cosh 2 \xi-\cos 2 \eta)^{2} t_{\eta}^{\eta}=\lambda(\cosh 2 \xi-\cos 2 \eta)\left(\frac{\partial^{2} u_{0}}{\partial \xi^{2}}+\frac{\partial^{2} u_{0}}{\partial \eta^{2}}\right)
$$$$
+(2 \mu+\kappa)\left[(\cosh 2 \xi-\cos 2 \eta)\left(\frac{\partial^{2} u_{0}}{\partial \eta^{2}}-\frac{\partial^{2} U}{\partial \xi \partial \eta}\right)\right.
$$$$
\left.+\sinh 2 \xi\left(\frac{\partial u_{0}}{\partial \xi}+\frac{\partial U}{\partial \eta}\right)-\sin 2 \eta\left(\frac{\partial u_{0}}{\partial \eta}-\frac{\partial U}{\partial \xi}\right)\right]
$$

$\frac{c^{2}}{2}(\cosh 2 \xi-\cos 2 \eta)^{2} t_{\eta}^{\xi}=\mu(\cosh 2 \xi-\cos 2 \eta)\left(2 \frac{\partial^{2} u_{0}}{\partial \xi \partial \eta}-\frac{\partial^{2} U}{\partial \xi^{2}}+\frac{\partial^{2} U}{\partial \eta^{2}}\right)$

$$
\begin{aligned}
& -(2 \mu+\kappa) \sin 2 \eta\left(\frac{\partial u_{0}}{\partial \xi}+\frac{\partial U}{\partial \eta}\right) \\
& -(2 \mu+\kappa) \sinh 2 \xi\left(\frac{\partial u_{0}}{\partial \eta}-\frac{\partial U}{\partial \xi}\right) \\
& -\kappa \frac{c^{2}}{2}(\cosh 2 \xi-\cos 2 \eta)^{2} \phi .
\end{aligned}
$$

Let $T^{\prime}$ denote the solution (2.6) with the boundary conditions:

(i) uniformly pressurised hole on $\xi=\xi_{0}$,

(ii) $t_{\xi}^{\xi}=t_{\eta}^{\xi}=m_{z}^{\xi}=0$ at $\xi=\infty$.

Let $\bar{T}$ denote the solution (2.6) with the undisturbed uniform field of uni-axial tension (i.e. as if there is no hole) $t_{x}^{x}=S$.

* Further details are available on request to the author. 
If $T$ denotes the required solution of the problem defined by (2.4) and (2.5) then we have

$$
T=T^{\prime}+\bar{T}
$$

The solution $\bar{T}$ is given by

$$
\begin{aligned}
& t_{\xi}^{\xi}=S \sinh ^{2} \xi \frac{1+\cos 2 \eta}{\cosh 2 \xi-\cos 2 \eta} \\
& t_{\eta}^{\xi}=-\frac{S}{2} \sinh 2 \xi \frac{\sin 2 \eta}{\cosh 2 \xi-\cos 2 \eta} \\
& t_{\eta}^{\eta}=S \cosh ^{2} \xi \frac{1-\cos 2 \eta}{\cosh 2 \xi-\cos 2 \eta} \\
& m_{z}^{\xi}=0
\end{aligned}
$$

We are only interested in the stress concentration factor $\left.t_{\eta}^{\eta}\right|_{\eta=\pi / 2}$ on the hole $\xi=\xi_{0}$, and this reduces to

$$
\left.t_{\eta}^{\eta}\right|_{\substack{\xi=\xi_{0} \\ \eta=\pi / 2}}=\left.S \cosh ^{2} \xi \frac{1-\cos 2 \eta}{\cosh 2 \xi-\cos 2 \eta}\right|_{\substack{\xi=\xi_{0} \\ \eta=\pi / 2}}=S .
$$

Similarly, using the solution $T^{\prime}$, the stress concentration factor reduces to

$$
\left.t_{\eta}^{\eta}\right|_{\substack{\xi=\xi_{0} \\ \eta=\pi / 2}}=\frac{2 S r_{2} / r_{1}}{1+M_{0}}
$$

where

$$
\begin{gathered}
M_{0}=\frac{\left(\frac{r_{2}}{r_{1}}\right)^{2}-1+32 d\left(1-\nu^{\prime}\right) \frac{b^{\prime 2}}{r_{1}^{2}}}{\frac{2}{r_{1}}\left(1+\frac{r_{2}}{r_{1}}\right)}, \\
d=1+\frac{2 \operatorname{Gek}_{2 n}\left(\xi_{0},-q_{1}\right)}{\operatorname{Gek}_{2 n}^{\prime}\left(\xi_{0},-q_{1}\right)}, \quad r_{1}=c \cosh \xi_{0}, \quad r_{2}=c \sinh \xi_{0}, \\
b^{\prime 2}=\frac{\gamma}{2(2 \mu+\kappa)}, \quad \nu^{\prime}=\frac{\lambda}{2 \lambda+2 \mu+\kappa},
\end{gathered}
$$

and the prime in Gek's denotes differentiation.

Using the complete solution $T$, the stress concentration factor is given by

$$
\left.t_{\eta}^{\eta}\right|_{\substack{\xi=\xi_{0} \\ \eta=\pi / 2}}=S\left(1+\frac{2 r_{2} / r_{1}}{1+M_{0}}\right)
$$




\section{Transition to circular case}

As the ellipse of semi-axes $r_{1}$ and $r_{2}$ tends to a circle with radius ' $a$ ', $\xi_{0} \rightarrow \infty, c \rightarrow 0$ such that $r_{1}, r_{2} \rightarrow a$ we get from (2.11)

$$
\left.t \eta\right|_{\substack{\eta=\pi / 2 \\ r_{1}, r_{2}=a}}=\frac{3+M_{0}}{1+M_{0}} S,
$$

which becomes $3 S$ in the classical case where $M_{0}=0$.

\section{Conclusion}

It is evident that the stress concentration factor proves to be smaller than in the classical theory (as $M_{0}>0$ ), depending on the elastic constants of the microstructure of the media. These results are important for solids composed of dumbell macro-molecules, such as fibrous and coarse grain structure materials.

\section{References}

[1] A. C. Eringen, 'Linear theory of micropolar elasticity', J. Math. Mech. 15 (1966), 909-923.

[2] S. Timoshenko and J. N. Goodier, Theory of Elasticity, 2nd Edition, McGraw-Hill (1951).

[3] S. Ghosh, 'Plane strain in an infinite plate with an elliptic hole', Bull. Cal. Math. Soc., Vol. XXVIII, No. 1 (1936), 21-47.

[4] H. Liebowitz (Ed.), Fracture, Vol. II (1968), 713-719.

[5] N. W. McLachlan, Theory and application of Mathieu functions, New York, Dover Publications (1964).

The University of New South Wales, Royal Military College, Duntroon, A.C.T., Australia. 